\title{
Electrical Conductance Time Constants for Freely Decaying Arcs
}

\author{
R. W. Anderson * and R. L. Phillips \\ University of Michigan, Ann Arbor, Michigan
}

Received April 2, 1973

\begin{abstract}
Electrical conductance time constants for the early stages of free decay after current modulation have been calculated from experimental measurements on a $5 \mathrm{~mm}$ diameter cascade arc at atmospheric pressure. The time constants were found by measuring the electric field response of the asymptotic portion of the arc column immediately after a sudden step decrease of arc current. The electric field strength was monitored by means of the copper cooling disks of the cascade, whose probe characteristics were studied thoroughly. The initial high current was supplied by a capacitor discharge circuit which was inductively compensated to produce a square wave pulse of $\sim 2 \mathrm{msec}$ duration. Time constants for initial decay were measured in both argon and nitrogen for initial currents ranging from 100 to 400 amperes. The initial free decay time constants of nitrogen were found to increase weakly from approximately 25 to $35 \mu \mathrm{sec}$ over the initial current range considered. The time constants of argon decreased from approximately 100 to $60 \mu \mathrm{sec}$ over the same initial current range.
\end{abstract}

\section{Introduction}

Recent theoretical and experimental results [1-14] have shown the initial variation of the electrical conductance in a cylindrical, wall stabilized, electric arc can be described by a time constant when the arc is subjected to a sudden change in current. All of the measurements $[6,9-12]$ which have been made to date, however, have been for small initial current levels (i. e. 100 amperes or less). The time constants obtained from these measurements have been theoretically predicted with a reasonable degree of accuracy by assuming the initial arc decay to be dominated by thermal conduction. This simplification is necessitated by the nonlinearity and coupling of the fundamental equations which describe the initial decay of the arc.

No theoretical analysis has investigated the combined effects of radial convection, radiation, variable pressure, and variable thermal diffusivity on the initial electrical conductance decay of an arc which is subjected to a sudden decrease in current. In addition, most analyses

* Presently on one year appointment with Gas Dynamics Laboratories, The University of Michigan, Ann Arbor, Michigan. The research reported here was supported by the National Science Foundation under Grant GK 14757 and is gratefully acknowledged. 
have studied only the conductance decay of a freely decaying arc; one whose current is suddenly discontinued. The analysis of Phillips [8] was an exception. Using a series solution technique for the temporal variation of the arc's properties, he discovered the initial arc decay to be one of constant density rather than constant pressure. He also showed the time constant for the initial conductance decay of a freely decaying arc is related to that for the initial decay of an arc whose current is step modulated. This result was later independently found by Graffman [9] using a perturbation technique with a small current step.

The result of Phillips' [8] theory has been to decrease the difficulty in making time constant measurements for the initial free decay of the electrical conductance in an arc, since now one can measure the time constant for the initial decay of the electrical conductance in an arc whose current is step modulated, and then simply relate it to the case of free decay. Measurements of this type should be more accurate than those made in the free decay case because the electric field strength can be continuously monitored by a well proven method $[15,16]$.

This paper presents results of time constant measurements for initial free decay made on a cascade arc [17] at atmospheric pressure using the current step modulation technique. These results greatly extend the available time constant data for the initial free decay of the electrical conductance in argon and nitrogen arcs with initial currents up to 400 amperes. These data should be useful for the further refinement of transient arc theories since they include the effects of radiation and radial flow on the decay of these initially high current arcs.

\section{Experiment}

\subsection{Cascade Arc}

A cascade arc, a schematic of which is shown in Fig. 1, is used as the arc confining device for this experiment. The cascade consists of a stack of water cooled copper disks which are insulated from each other by aluminum disks that are dielectrically anodized. Each copper disk has a concentric hole of $5 \mathrm{~mm}$ diameter such that a well cooled cylindrical chamber is formed by the stack. The cooled copper disks are $6.35 \mathrm{~mm}$ thick and are separated from each other by $1.60 \mathrm{~mm}$. A teflon O-ring on either side of the aluminum disks provides a gas tight and an electrically nonconducting seal between the cooling disks. The disks are held together by end plates each of which has a removable viewing cap and inlet gas ports. The entire arrangement is gas tight, capable of operating above or below atmospheric pressure.

Electrodes are located at each end of the cascade column. The anode is water cooled copper while there are four equally spaced cathodes 


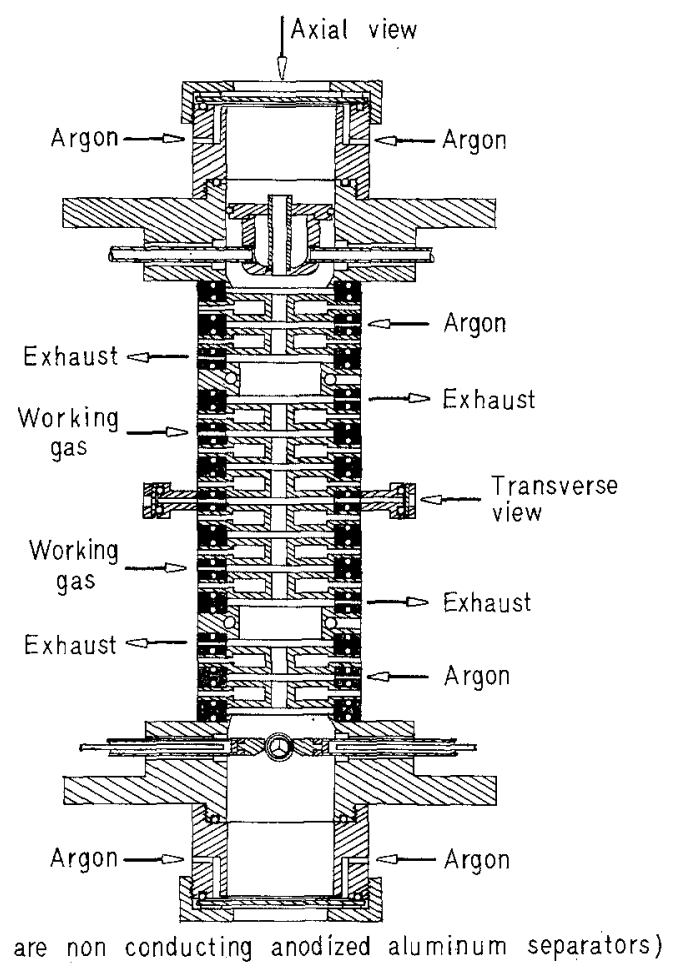

Fig. 1. Schematic cross-section of cascade chamber

arranged perpendicular to the arc column. The cathodes are water cooled and constructed of thoriated tungsten. The electrode regions are continually bathed with argon, while either nitrogen or argon is used as the working gas. The plasma expansion regions at each end of the column help to prevent any working gas from reaching the electrodes.

The argon and nitrogen gases were of standard commercial purity. When nitrogen was used as a working gas, the arc was continuously monitored for the presence of any argon [18]. It was possible to ensure that the purity of the nitrogen in the working gas column was greater than $99 \%$ prior to the making of any measurements.

The arc was capable of operating at steady current levels up to 200 amperes and was operated at atmospheric pressure in all cases.

\subsection{Pulse System and Application}

In order to attain the initially high currents which were necessary to make the time constant measurements, the current pulse system shown 


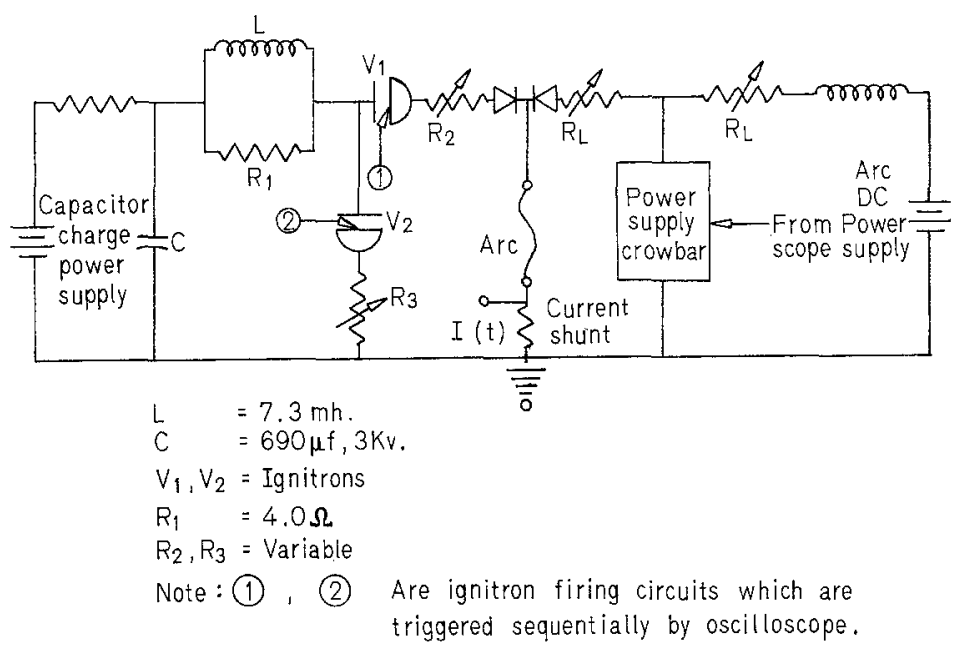

Fig. 2. Circuit used to produce square wave current pulse

in Fig. 2 was developed. It is comparable to the circuit proposed by Durnford and McCormick [19]. The system was designed to produce a variable current, square wave pulse with a maximum pulse current of 500 amperes. The pulse duration is approximately $2 \mathrm{msec}$. The variation of the peak current was always less than $3 \%$.

The resistance $R_{2}$ was varied between 2 and 8 ohms, depending on the capacitor charge and the maximum current desired. The resistance $R_{3}$ was adjusted so the arc did not extinguish after the pulse. Its value was typically near $1 \mathrm{ohm}$.

The ignitrons were triggered by standard methods [18]. They were found to break down in less than $2 \mu \mathrm{sec}$ when their initial anode voltages were greater than 800 volts. This placed a lower limit on the capacitor charge voltage of approximately 1000 volts, which resulted in a minimum current pulse of approximately 100 amperes.

The function of the diodes is to isolate the DC power supply and pulse system from each other. Since they have no time constant associated with their duration of voltage standoff, they ensure a longer measurement time than in the case where an inductor is used to isolate the power supply [9]. The diode bank isolating the power supply from the pulse system had a peak inverse voltage rating of 2000 volts and a forward current rating of 40 amperes. Due to its fast response to downstream impedance changes, the current regulated power supply had to be short circuited into an initially uncharged capacitor bank during the current pulse to completely eliminate any of its effects on the time constant measurements [18]. 


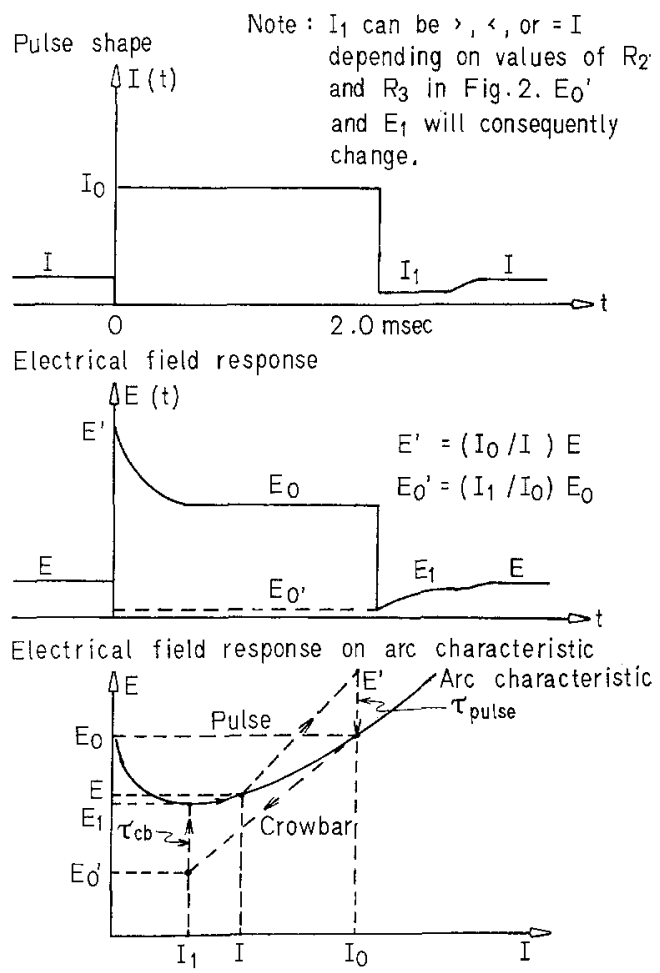

Fig. 3. Schematic of arc pulse response

The arc, which was initially operating at a low DC current, was pulsed with the triggering of ignitron $V_{1}$. After the arc had thermally readjusted to its new level, ignitron $V_{2}$ was triggered which caused the arc current to suddenly decrease to a lower steady state level. The time constants were measured during the time immediately after the crowbarring of the current pulse.

A schematic of the anticipated arc response to the square wave current pulse is shown in Fig. 3. The period of interest for the measurement of the electrical conductance decay is during the time the electric field strength changes from $E_{0}^{\prime}$ to $E_{1}$, or the time labeled as $\tau_{c b}$ on the bottom figure. Since the electrical conductance is equal to the ratio of the arc current divided by the electric field strength, all one needs to do is measure these two quantities to find a temporal history of the decay.

The transient arc current was found by measuring the voltage drop across a carbon rod shunt whose resistance was precisely measured on a wheatstone bridge [18]. The transient electric field response of the 
arc to the current pulse was measured by using the water cooled copper disks of the cascade arc as electrical probes $[15,16]$. Since the use of these disks for the electric field measurements is nontrivial, an investigation of their electrical characteristics was essential.

\subsection{Probe Characteristics}

Maecker [15] first used the cascade disks as electrical probes to measure the electric field strength of the arc. The electric field strength was found by dividing the voltage difference between two cascade disks by their geometric separation. He suggested drawing an equal electron current from each probe to establish good electrical contact with the adjacent plasma.

Edels and Kimblin [16], however, were the first to study the probe characteristics of the cascade disks and give valid physical reasoning for Maecker's suggestion. They were able to show that serious errors in the arc electric field strength could occur if one simply measured the voltage difference between two probes. An explanation for this error was found by studying the voltage-current characteristic of each probe. The voltage-current characteristic of each probe was found by measuring the current response of the probe to a series of impressed voltages on the probe while the arc was operating at a steady current. A schematic of two of these probe characteristics is shown in Fig. 4. The electron current portions of the characteristics of the probes were found to have equal slopes while the ion current portions were not. The ion current portion of each characteristic was observed to approach the zero current axis as the arc current decreased. The point of zero current on each electrical characteristic of the probes was defined as the point of floating potential. The voltage measured there is that value which would be

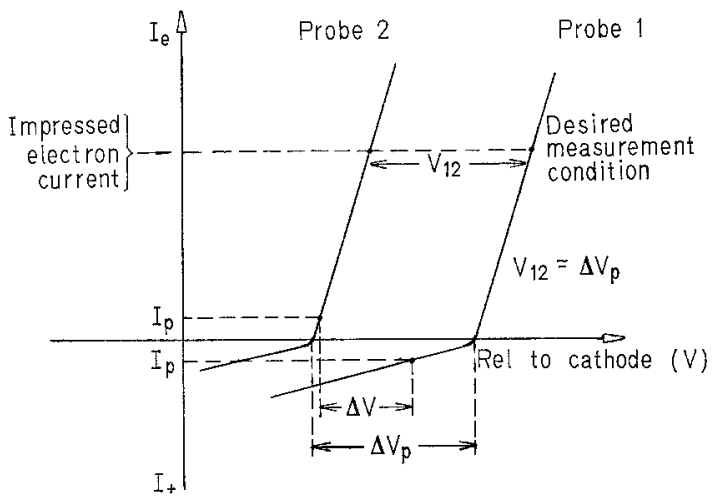

Fig. 4. Schematic of probe pair voltage-current response 
measured at any cascade disk if it was isolated from the adjacent cascade disks by an infinite impedance; this is the value one wishes to measure. However, since there is a finite impedance between the probe pair, an interprobe current, $I_{p}$, flows which causes their measured voltage difference, $\Delta V$, to be in error. This explains the reason for drawing an equal electron current from each probe, since the parallel region of the electron current portion of each of the characteristics extends through the point of floating potential, thereby equating $V_{12}$ with $\Delta V_{p}$.

By electrically connecting two adjacent probes, Edels and Kimblin also showed the probes assume the plasma potential adjacent to their lowest potential edges. The method of current collection of the probes was discovered to be diffuse, rather than that due to localized spot collection. Thus the finite probe thickness caused it to collect a net electron current at voltages lower then the floating potential [20].

One therefore needs to know the probe characteristics for a given cascade before using a probe pair to make reliable column voltage gradient measurements. Primarily, one must be certain that the electron current portions of the probe characteristics have equal slopes through their respective points of floating potential up to the value of electron current drawn from the pair. The single probe characteristics can also tell one the maximum value of electron current which can safely be drawn without causing the probe plasma sheath to break down [20].

Rather than measure the characteristic of each probe, a point at. a time, at a given current level [16], the characteristics were found dynamically $[18,20]$. With the arc operating at constant current, the voltage of a probe is raised to its point of floating potential. An AC voltage is then superimposed on the floating potential voltage of the probe. The resulting voltage-current response of the probe is then recorded oscillographically. This method is repeated for all of the probes of interest. These probes are normally those in the asymptotic portion of the arc column where the electric field strength is constant and independent of any electrode effects [21].

Examples of probe characteristics of an argon arc are shown in Fig. 5. The probes used are the centermost copper disks which are shown schematically in Fig. 1. These characteristics were measured with a Tektronix 565 oscilloscope equipped with a 3 A 3 vertical preamplifier. The electron portions of the characteristics are seen to be parallel. The ion current portion of the characteristics is seen to dip further below the point of floating potential as the arc current increases, thereby necessitating the drawing of less electron current to make reliable probe measurements of the electric field strength.

Probe characteristics measured when the arc gas was nitrogen were found to be similar to those observed for argon [18]. The primary differ- 


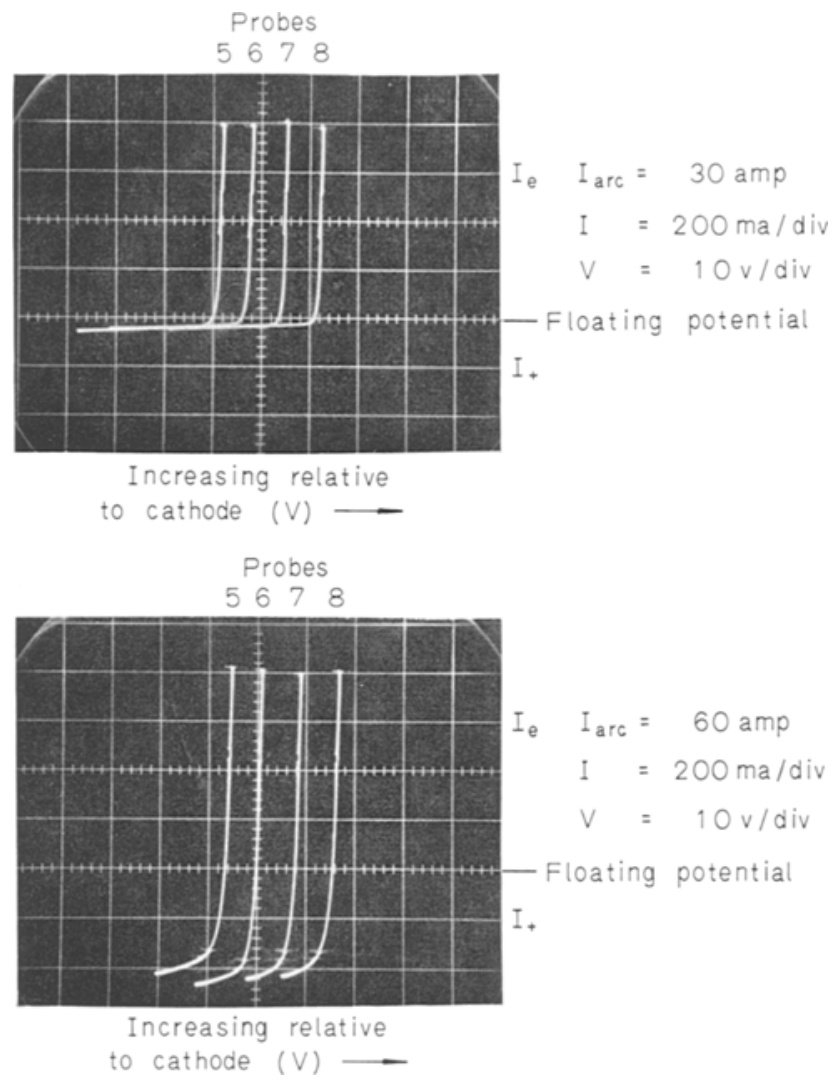

Fig. 5. Examples of dynamic probe characteristics in argon

ence was in the ion portion of the characteristics, which was found to lie closer to the point of floatening potential in the case of nitrogen at the same arc current level. This is thought to be due to the more constricted nature of the nitrogen arc, which would have a thicker and less conducting probe sheath at a given current than that in an argon arc.

When using a probe pair to measure transient changes in the electric field strength, one must also consider the effects of any interdisk capacitance between them [16]. In the case of this experiment, when the electric field strength falls very fast after the sudden decrease in arc current, an interprobe current flows due to the discharging of the interprobe capacitance of the probe pair. The time constant associated with this discharge is equal to the product of the interprobe capacitance and the sum of the individual probe-to-plasma contact resistances. The effects of this discharge time on the electric field measurements can be appreciable when one simply connects to the probes and measures the resulting voltage 
difference. This is due to the high resistance associated with the ion current contact to the plasma of the higher potential probe of the pair. This effect is minimized when electron current is drawn from each probe of the pair.

Probes 5 and 7 were used to measure the electric field strength. The interprobe resistance was measured as approximately $300 \mathrm{~K}$ ohms. The interdisk capacitance was found to be approximately 20 picofarads. To minimize capacitance effects, the measuring probes were biased to the arc anode, such that they each drew approximately 50 milliamperes of electron current when the arc anode was 100 volts above cathode potential. A further check on probe capacitance effects was made by inserting a capacitance 2 orders of magnitude greater than that measured between the probe pair and measuring the electric field response to a given pulse. No significant change was recorded.

\section{Method of Data Reduction}

The time constants for initial free decay of the conductance were deduced with the aid of a recent theory of Phillips [8]. This theory allows one to relate the time constant of an interrupted arc (free decay) to the conductance time constant of a step modulated arc. As noted, measurements on a step modulated arc are easier to carry out since there always exists an electric field which one can monitor. The significant relations for obtaining time constant data for the conductance decay are derived as follows.

The initial variation of the conductance immediately after step modulation relative to an arbitrary conductance $G_{1}$ can be found in Ref. [8], Eq. (10) as

$$
G(t)-G_{1}=G_{0}-G_{1}+2 \pi R^{2} E_{0}^{2}\left\{\left(\frac{I_{1}}{I_{0}}\right)^{2}-1\right\} K t+O\left(t^{2}\right)
$$

where $G_{0}$ is the initial conductance (which is equal to $I_{0} / E_{0}$ ), $I_{1}$ is the final current, and $K$ is a constant which results from a complicated integral of the initial arc state.

The time constant for the variation of this differential conductance can be written as

$$
\left[\frac{d \ln \left\{G(t)-G_{1}\right\}}{d t}\right]_{t=0}=\tau_{g}^{-1}=\frac{2 \pi R^{2} E_{0}^{2}}{G_{0}-G_{1}}\left\{\left(\frac{I_{1}}{I_{0}}\right)^{2}-1\right\} K .
$$

The time constant for the initial free decay of the conductance can be found from Eq. (1) to be

$$
\left[\frac{d \ln G(t)}{d t}\right]_{t=0}=\tau_{g_{\mathrm{f}, \mathrm{d}}}^{-1}=\frac{-2 \pi R^{2} E_{0}^{2} K}{G_{0}} .
$$


The relation between the time constant for the initial free decay of the conductance and time constant for the initial variation of the differential conductance after step modulation can now be written as

$$
\tau_{g_{\text {f. d. }}}=\frac{G_{0}}{G_{0}-G_{1}}\left[1-\left(\frac{I_{1}}{I_{0}}\right)^{2}\right] \tau_{g} .
$$

The time constant for the initial variation of the differential conductance after step modulation can be found by plotting $G(t)-G_{1}$ vs. $t$ on a semilog graph. If the initial conductance decay can be approximated by a straight line, its slope is related to the time $\tau_{g}$ by

$$
\tau_{g}=\frac{\left(t_{2}-t_{1}\right) \log _{10} e}{\log _{10}\left\{\frac{G\left(t_{1}\right)-G_{1}}{G\left(t_{2}\right)-G_{1}}\right\}}
$$

where $G\left(t_{1}\right)-G_{1}$ and $G\left(t_{2}\right)-G_{1}$ are any two values on the linear approximation to the initial $\left(G(t)-G_{1}\right)$ differential conductance decay for $t_{2}>t_{1}$. In practice, $G_{1}$ was taken as the final conductance value at $I_{1}, t_{1}$ was always taken as zero, and $t_{2}$ was taken as the time when $\left(G\left(t_{1}\right)-G_{1}\right) /$ $\left(G\left(t_{2}\right)-G_{1}\right)=10$ on the straight line approximation. The time constant for the initial decay of the differential conductance can then be written as

$$
\tau_{g}=0.434 t_{2} .
$$

The time constant for the initial free decay of the conductance is then easily calculated with the aid of Eq. (4).

An example of the method of data reduction for the time constant of the initial decay of the differential conductance in an argon arc whose current was step modulated is shown in Fig. 6. A Tektronix 531 A oscilloscope with a type $\mathrm{G}$ differential vertical amplifier was used to record the transient current. An average value was accepted for the final current, which was nominally the value at the midpoint of the total adjustment time. A Tektronix 7503 oscilloscope with a type 7A 22 differential vertical amplifier was used to record the transient differential voltage response of the probes. Compensating probes were used with the amplifier to minimize the voltage error. The DC offset feature of this differential amplifier allowed one to make the probe response measurements on a lower input voltage scale, thereby increasing the measurement sensitivity.

Results of data reduction for a nitrogen arc whose current was step modulated are qualitatively similar to those found for an argon arc, the only exception being the magnitude of the time constants which are smaller. 

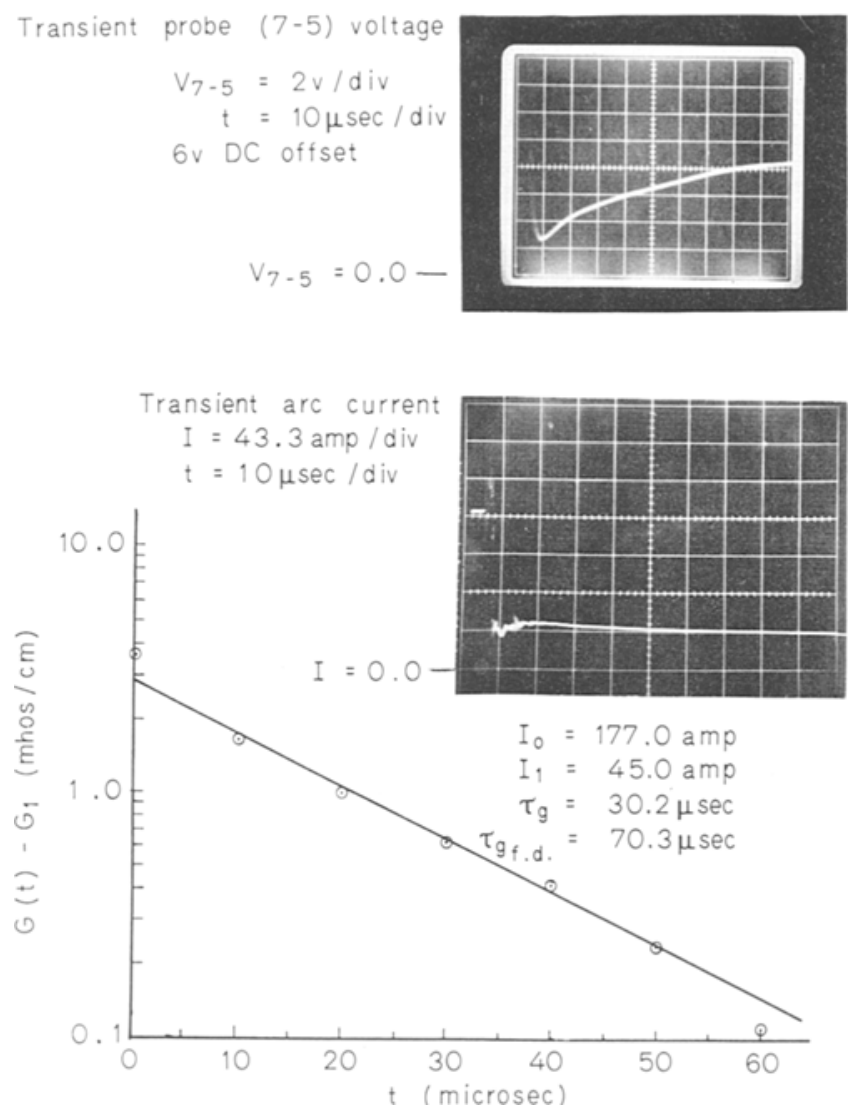

Fig. 6. Example of time constant reduction for pulsed argon arc

\section{Results and Discussion}

Results of the time constant measurements are shown in Fig. 7 for argon and Fig. 8 for nitrogen. The lower current limit on both sets of data was due to limitations of the pulse system. The upper current limit was established by an erratic electric field response from the probes. A time lag in the maximum of the electric field response immediately after the crowbarring of the current pulse was also evident for the high current data ( $>400$ anperes). These results were therefore excluded.

The final electric field strength for the argon data was within $25 \%$ of that measured at the same final current in the steady state. This value was $20 \%$ for the nitrogen data. This result could possibly contribute to the larger scatter of the argon data. One would not think this to be the case, however, since the effect of this result enters into the time constant deri- 


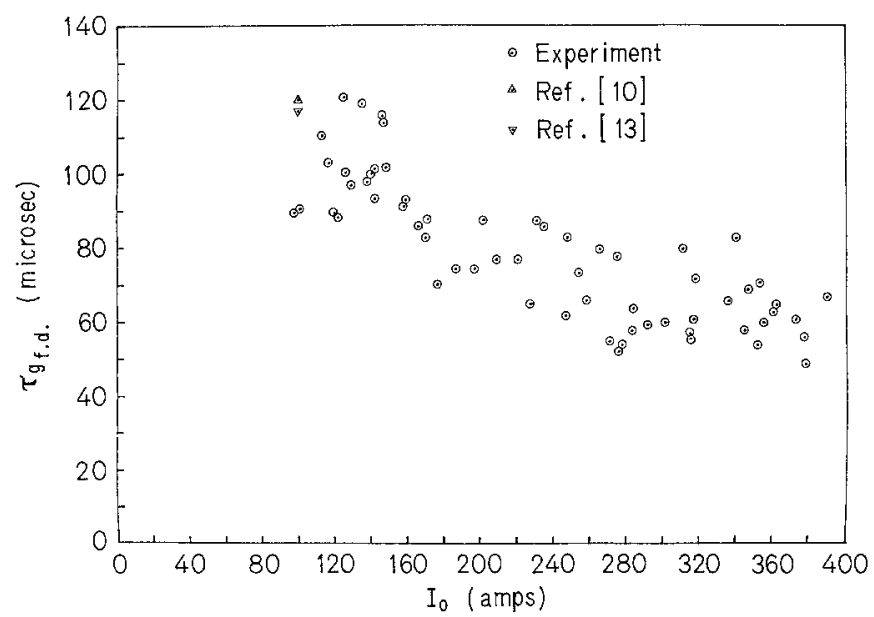

Fig. 7. Conductance time constants for initial free decay in argon vs. initial current

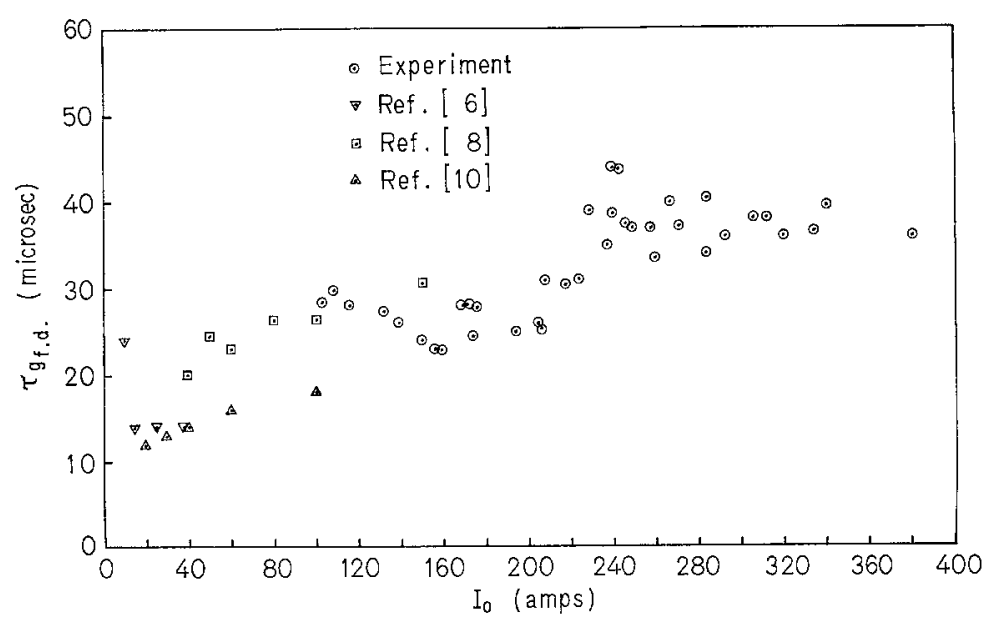

Fig. 8. Conductance time constants for initial free decay in nitrogen vs. initial current

vation through the arbitrary conductance value $G_{1}$. The important parameter is the rate of change of conductance immediately after step modulation.

The lack of any other high current time constant data makes it difficult to judge the accuracy of these measurements. For argon, the experimental result [10] and the theoretical result [13] are slightly higher than those which were measured. The theoretical result does not account for all of the energy loss mechanisms which would undoubtedly lower the time 
constant value while the method of measuring the conductivity decay in Ref. [10] is most likely subject to some errors. If one accepts the scatter of the argon data presented herein as its error bound, the error bounds of the data at 100 amperes would undoubtedly fall within each other.

The time constants for the inital free decay of the conductance of the argon arc are seen to decrease with increasing arc current. This is in contrast to the increasing time constants which would be predicted by simple arc theory, thereby indicating the necessity for considering more energy loss mechanisms in the theoretical analysis of the initial decay of high current arcs.

The time constants for the initial free decay of the nitrogen arc agree much better with the available experimental $[6,10]$ and theoretical [8] results than those found for argon. If one accepts the error bound of the data as the scatter, the results agree very well with those of Phillips [8] while the result of Ref. [10] is slightly lower at 100 amperes. This discrepancy might again be partially attributed to the method which they used to measure the conductance decay.

The time constants for the nitrogen arc increase weakly with current and would appear to be predicted better by simple arc theory than those for a comparable current argon arc. This result is expected if one compares the total emitted radiation from the two gases at the same current level [18]. The conducting radius of the nitrogen arc is also more definable and observed to increase with increasing current level.

Other effects which were not measurable could also affect the accuracy of the results. The effects of any occurrence of radial convection immediately after the crowbarring of the current could have altered the probe measurements. The time constants could have also been measured during a period of axial pressure relief. The sudden energy addition of the pulse to the arc will cause it to expand axially. It is not known if the arc had thermally adjusted completely to its new current level before it was crowbarred to the lower current level. However, the electric field strength immediately before crowbarring was found to be within $10 \%$ of that which was measured at steady state conditions. The effects of axial pressure relief, if occurring, will be more pronounced with increasing pulse current.

Deviations from local thermodynamic equilibrium within the decaying arc $[12,18]$ could also be occurring which could create discrepancies between the experimental and theoretical results.

Finally, one should consider that although these results were compared with other data for a $5 \mathrm{~mm}$ diameter arc there will always be discrepancies in the results, since the diameter is usually the only geometric similarity these arcs have in common. The effect of transients of the types we have considered could only be to increase any discrepancies due to geometric dissimilarities between experiments. 


\section{Conclusions}

We conclude from the results of the preceeding work that time constants measured by crowbarring a high current pulsed arc must be critically examined. This is because axial and radial pressure adjustment might not occur fast enough that a quiescent plasma state is reached within the arc column at the time of crowbar. Thus, before crowbarring, the arc column could initially be at a higher or lower pressure than anticipated. To measure in detail any pressure effects in the arc would require a great deal of effort, due to the fast response times needed, the complicated arc containment geometry, and the large amount of radiation present.

The concept of a time constant for a particular gas is useful to circuit breaker designers as a breaker figure of merit. One of the aims of this study was to find conductance time constants of gases isolated from flow effects. It was found that flow effects as well as radiation effects could be very important to the decay of initially high current arcs. It therefore appears that the time constants found in cascade arcs will be of limited usefulness in predicting a gas interrupting capability, except on a relative basis. This suggests that one should theoretically and experimentally study the complete transient arc-nozzle flow problem to understand how all the nonlinearities of the problem are interrelated. Such an investigation is presently being undertaken at this laboratory.

\section{References}

1. Frind, G.: Z. Angew. Phys. 12, 5, 231 (1960); 12, 11, 515 (1960)

2. Edels, H., Fenlon, F. H.: Proc IEEE 110, 11, 2082 (1963)

3. Phillips, R. L.: Proc. IEEE 113, 4, 117 (1966)

4. Edels, H., Fenlon, F. H.: Brit. J. Appl. Phys. 16, 219 (1965)

5. Jones, G. R., Edels, H.: Z. Physik 229, 14 (1969)

6. Hearne, K. R., Rasul, M.: Brit. J. Appl. Phys. 1, 753 (1968)

7. Jones, G. R., Freeman, G. H.: Z. Physik 229, 177 (1969)

8. Phillips, R. L.: Z. Physik 211, 113 (1968)

9. Graffman, E.: Dissertation, Univ. Liverpool, England (1967)

10. Christmann, H., Frie, W., Hertz, W.: Z. Physik, 203, 372 (1967)

11. Hertz, W.: Z. Physik 245, 105 (1971)

12. Christmann, H., Hertz, W.: Proc. 7 Intern. Conf. Phen. Ionized Gases, 1, 769 (1965)

13. Detloff, L.: Dissertation, Rheinisch-Westfälischen Tech. Hoch. Aachen, (1969)

14. Holmes, R., Jones, G. R.: Z. Physik 215, 466 (1968)

15. Maecker, H.: Z. Physik 158, 392 (1960)

16. Edels, H., Kimblin, C. W.: Brit. J. Appl. Phys. 17, 1607 (1966)

17. Maecker, H.: Z. Naturforsch. 11 a, 457 (1956)

18. Anderson, R. W.: Dissertation, Univ. Mich., Ann Arbor, 1972

19. Durnford, J., McCormick, N. R.: Proc. IEE 99, 67, 33 (1952)

20. George, D. W., Richards, P. H.: Brit. J. Appl. Phys. 1, 2, 1171 (1968)

21. Uhlenbusch, J., Gieres, G.: Z. Angew. Phys. 27, 66 (1969)

Associate Professor Richard L. Phillips and Dr. Richard W. Anderson. The University of Michigan. Department of Aerospace Engineering. Ann Arbor, Michigan, USA. 